\title{
DETERMINATION OF ABSOLUTE FISSION CROSS SECTION RATIOS USING THE METHOD OF THRESHOLD CROSS SECTIONS
}

\author{
James W. Behrens
}

Novcuiber 12, 1973

Prepared for U.S. Atomic Energy Commission urder contract No. W-7405-Eng-48

\section{MASTER}



TID-4500, UC-34c

Physics - Nuclear

\title{
니 \\ LAMTENCE LNEFMORE LAEORATOFY

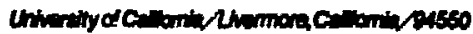

\section{UCRL-51478 \\ DETERMINATION OF ABSOLUTE FISSION CROSS SECTION RATLOS USING THE METHOD \\ OF THRESHOLD CROSS SECTIONS}

\author{
James W. Behress
}

MS, date: November 12, 1973

Norice

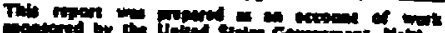

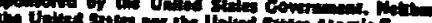

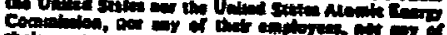

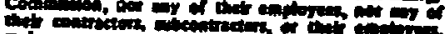

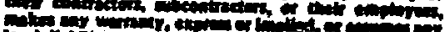

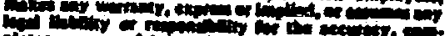

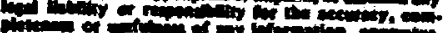

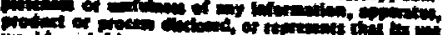

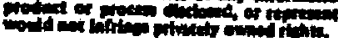




\title{
DETERMINATION OF ABSOLUTE FISSION CROSS SECTION RATIOS USING THE METHOD OF THRESHOLD CROSS SECTIONS
}

\begin{abstract}
Fission cross section measurements for a collection of uranium and plutonium isotopes are presently being made at LLL using he 100-MeV electron linear accelerator as a pulsed source for neutrons. The cross sections are measured as a function of neutron energy from $15 \mathrm{MeV}$ down to thermal energy using the time of night technique.

For neutron energies above $1 \mathrm{MeV}$, we use a new method for determining absolute fission cross section ratios. This method, which we call the method of threshold cross sections, gives absolute fission cross section ratios without the need for determining the absolute masses of fissionable material within our fission chambers.

An explanation of the threshold cross section method is given and an example is cited for the absolute ratio $\sigma_{f}(238): \sigma_{f}(235)$ above $1 \mathrm{MeV}$.
\end{abstract}

\section{Introduction}

Fission cross section measurements for the uranium and plutonium isotopes have been made by many people over the past decades, and, when their results are compared, one finds large disagreements between the published data sets.

In making fission cross section ratio measurements a common approach has been to use back-to-back fission chambers on which the same neutron nus is incident on both fission chambers and the absolute fission cross section ratio can be determined once the absolute mass of fissionable material is known for each fission chamber. This mass analysis technique generally has an uncertainty of $\sim 1 \%$. By using the method of threshoid cross sections the need for absolute mass determination is eliminated and one only needs to know the isotopic atom percents of the fissionable isotopes in each fission chamber. Using mass spectrographic methods, these atom peicents can be determined with uncertainties of $<0,1 \%$.

\section{The Method of Threshold Cross Sections}

The method of threshold cross sections takes advantage of the rapid falloff in cross section for a threshold reaction below the threshold energy. The method will be 
illustrated by an example in which I will cite the neutron fission reaction, i.e., the ( $n, f)$ reaction, in the uranium isotopes ${ }^{235} \mathrm{U}$ and ${ }^{238} \mathrm{U}$. The ${ }^{235} \mathrm{U}(\mathrm{n}, \mathrm{f})$ cross section extends from high neutron energy all the way down to thermal energy. The ${ }^{238} U(n, f)$ cross section, however, drops off sharply at $E_{n}<1 \mathrm{MeV}$ and only the small subthreshold fisBion cross section remains. Our goal is now to measure the absolute ratio $\sigma_{f}(238)$ : $\sigma_{f}(235)$ by the thresinold cross section method.

Two fission chambers in a back-to-back configuration are required. The first fission chamber (FC-1) will be referred to as the composition fission chamber since it contains uranium having $\alpha$ at.\% ${ }^{235} U$ and $\beta$ at.\% ${ }^{238} U$. Here high purity ${ }^{235} U$ and ${ }^{238} U$ should be mixed to produce the desired atom percents. Thi second fission chamber (FC-2) contains high purity ${ }^{235} \mathrm{U}$ only. The fission foils crin be produced by a painting techaique to minimize waste of the fissionable material.* Mass spectrographic methods can be used to determine the uranium isotopic atom percents with an uncertainty of $<0.1 \%$.

The back-to-back fission chambers are exposed to the neutron spectrum from the LLL $100 \mathrm{MeV}$ electron linear accelerator, which provides a pulsed source of neutrons. The neutron time of flight method to determine the ratio as a function of neutron energy is used at the 34-meter time-of-flight station on the 259-meter time-of-night tube of the accelerator.

The fission rate as a function of neutron energy in a fission chamber can be expressed by

$$
\begin{aligned}
& N\left(E_{n}\right) \\
& \text { Fissions/sec }
\end{aligned}=N_{A} \sigma_{f}\left(E_{n}\right) \phi .
$$

For the composition fission chamber (FC-1) the fission rate is

$$
\underset{\text { Fissions/sec }}{N\left(E_{n}\right)}=\left(\frac{\alpha}{100}\right) \underset{F C-1}{N_{A}} \quad 235 \sigma_{f}\left(E_{n}\right) \phi+\left(\frac{\beta}{100}\right) \underset{F C-1}{N_{A}}{ }^{238} \sigma_{f}\left(E_{n}\right) \phi .
$$

For the high purity ${ }^{235} \mathrm{U}$ fission chamber (FC-2) the fission rate is

$$
\begin{aligned}
& N\left(E_{n}\right) \\
& \text { Fisions/sec } \\
& \text { FC-2 }
\end{aligned}
$$

The ratio of these two rates is then

\footnotetext{
${ }^{*}$ J. W. Behrens, Preparation of Fission Foils for Fission Ionization Chambers Using a Painting Technique, Lawrence Livermore Laboratory, Rept. UCRL-51476 (1973). 


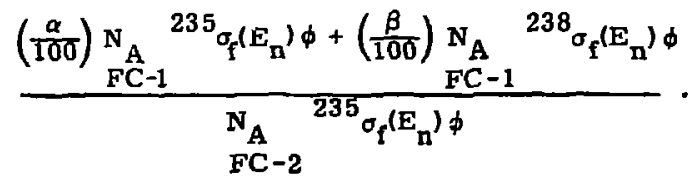

Since both fission chambers see the same incident neutron flux the ratio becomes

$$
\frac{\left(\begin{array}{c}
\alpha \\
100
\end{array}\right){ }_{F C-1}{ }^{235} \sigma_{f}\left(E_{n^{\prime}}+\left(\frac{\beta}{100}\right) N_{A}{ }^{238} \sigma_{f}\left(E_{n}\right)\right.}{N_{A}{ }^{235} \sigma_{f}\left(E_{n}\right)}
$$

This ratio is a function of neatron energy but at $E_{n}<1 \mathrm{MeV}$ the ${ }^{238} \mathrm{U}(\mathrm{n}, \mathrm{f})$ cross section is essentially zero compared to the ${ }^{235} U(n, f)$ cross section. Therefore for $E_{n}<1 \mathrm{MeV}$ the ratio is a constant equal to

$$
\frac{\left(\frac{\alpha}{100}\right) N_{A}}{N_{A} C-1}
$$

The ratio can be multiplied by the inverse of this constant. The result is

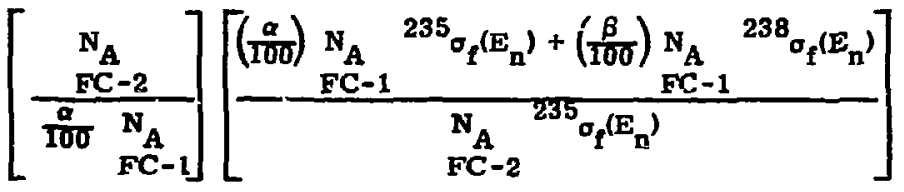

$$
\begin{aligned}
& =1+\frac{\sigma}{\alpha}\left[\frac{{ }^{238} \sigma_{f}\left(E_{n}\right)}{235 \sigma_{f}\left(E_{n}\right)}\right] \text {. }
\end{aligned}
$$

Since $\beta / \alpha$ is known, you are left with an expression containing the absolute fission crosg section ratio $\sigma_{f}(238): \sigma_{f}(235)$. By including a third fiseion chamber containing only high purity ${ }^{238} \mathrm{v}$ in the same back-to-back configuration, the relative ratio $\sigma_{f}(238)$ 0 (235), obtained by ratioing the high purity ${ }^{238} \mathrm{U}$ fission chamber to the high purity ${ }^{235} \mathrm{U}$ ngsion chamber, can be normalized to the absolute ratio and extended to $E_{n}<1 \mathrm{MeV}$ where $\sigma_{l}(238) \ll \sigma_{f}(235)$.

In a similar manner the threshold cross section method can be used to determine such absolute ratiog as $\sigma_{f}(234): \sigma_{f}(235), \sigma_{f}(236): \sigma_{f}(235)$, and $\sigma_{f}(238): \sigma_{f}(233)$. In the last case $1 /\left[\sigma_{f}(238): \sigma_{f}(233)\right]$ multiplied by $\sigma_{f}(238): \sigma_{f}(235)$ gives $\sigma_{f}(233): \sigma_{f}(235)$. 